\title{
The CST Bounce Universe model - A Parametric Study
}

\author{
Yeuk-Kwan E. Cheung ${ }^{1,2^{*}}$, Xue Song ${ }^{1,3}$, ShuYi Li ${ }^{1,4}$, YunXuan $\mathrm{Li}^{1,5}$, and YiQing Zhu ${ }^{1,6}$ \\ ${ }^{1}$ Department of Physics, Nanjing University, Nanjing 210098, China; \\ ${ }^{2}$ Institute of High Energy Physics, Beijing, China; \\ ${ }^{3}$ Department of Physics, Princeton University, Princeton NJ 08544, USA; \\ ${ }^{4}$ Department of Physics, Brown University, Providence RI 02912, USA; \\ ${ }^{5}$ California Insitute of Technology, Pasadena CA 91125, USA; \\ ${ }^{6}$ ETH Zürich, 8092 Zürich, Switzerland
}

Received April 6, 2018; accepted May 22, 2018; published online June 15, 2018

\begin{abstract}
A bounce universe model with a scale-invariant and stable spectrum of primordial density perturbations was constructed using a consistent truncation of the D-brane dynamics from Type IIB string theory. A coupling was introduced between the tachyon field and the adjoint Higgs field on the D3-branes to lock the tachyon at the top of its potential hill and to model the bounce process, which is known as the Coupled Scalar and Tachyon Bounce (CSTB) Universe. The CSTB model has been shown to be ghost free, and it fulfils the null energy condition; in addition, it can also solve the Big Bang cosmic singularity problem. In this paper we conduct an extensive follow-up study of the parameter space of the CSTB model. In particular we are interested in the parameter values that can produce a single bounce to arrive at a radiation-dominated universe. We further establish that the CSTB universe is a viable alternative to inflation, as it can naturally produce a sufficient number of e-foldings in the locked inflation epoch and in the post-bounce expansion to overcome the four fundamental limitations of the Big Bang cosmology, which are flatness, horizon, homogeneity and singularity, resulting in a universe of the current size.
\end{abstract}

bounce universe, big bang singularity, tachyon inflation, flatness and horizon problems

PACS number(s): $11.25 . \mathrm{Uv}, 11.25 . \mathrm{Mj}, 11.25 . \mathrm{Wx}, 98.80 . \mathrm{k}, 98.80 . \mathrm{Cq}$

Citation: $\quad$ Y.-K. E. Cheung, X. Song, S. Y. Li, Y. X. Li, and Y. Q. Zhu, The CST Bounce Universe model - A Parametric Study, Sci. China-Phys. Mech. Astron. 61, 000000 (2018), doi: 10.1007/s11433-018-9251-0

\section{Introduction}

The four most fundamental problems in the Big Bang cosmology are Flatness, Horizon, Homogeneity and Singularity. In the 1980s inflation was proposed [1] (with an update in [2]) to address the first three of these problems. The predictions of the inflation paradigm are consistent with the high-precision cosmological observations, such as the the highly isotropic and homogeneous cosmic microwave background radiation (CMBR) [3], and the scale invariance of primordial density perturbations attributed to the quantum fluctuations of the inflaton field at the end of inflation [4]. Inflation, however, has its problems. For instance, the initial singularity does not dis- appear but is delayed [5].

Building on the success of inflation models, cosmologists have been searching for alternative scenarios that can address the problem of the cosmic singularity. In 1993 a GRcompatible solution with a nonsingular bounce was proposed shortly after the first observation of current expansion [6]. Meanwhile string theorists attempted to implement a nonsingular bounce in dilaton gravity, leading to the pre-big-bang (PBB) scenario within the string theory framework [7]. A period of intensive string-inflation model building ensues, as reviewed by Linde [8], and Tye [9].

The bounce universe itself has a long history, and many ingenious models are reviewed in [10]. A cyclic model in a con- 
ventional four-dimensional quantum field theory generates a bounce that solves the singularity problem [11]. "Matter bounce" postulates a period of contraction, in which the scale invariant spectrum of primordial density perturbations is generated $[12,13]$, prior to the Big Bounce; at the Big Bounce the universe turns around and enters a period of expansion resulting in our existing universe. Explicit model building follows. Ghost condensation was proposed to avoid ghosts in the perturbation spectrum [14-18]. $N=1$ supergravity is used in a bounce model to eliminate ghost excitations [19] and to prove that nonsingular bounces were viable in supergravity. "G-Bounce" models generate a non-singularity inflation cosmos with a Galileon field [20], while Lee-Wick bounce produces a bounce in the radiation phase with an apparent scale invariance in the perturbation spectrum [21,22]. A bounce solution is also found in the universe dominated by the Quintom matter $[23,24]$. Furthermore the well-known Ekpyrotic universe [25] is inspired by D-branes dynamics. Bounce conditions were also found possible in $f(R)$ cosmology [26].

Encouraged by these advances in bounce cosmology we improved on the original tachyon inflation model proposed independently by Sen [27, 28] and Gibbons [29], by incorporating an interaction between the tachyon fields and adjoint Higgs fields. Thus we can not only we greatly improve the number of e-foldings obtained from the model by locking the tachyon at the top of its potential hill with the Higgs fields, but also realize a bounce universe in string theory with this consistent truncation of the D-brane dynamics. We call this the coupled scalar-tachyon bounce universe model or CSTB for short [30-32].

The CSTB model has several salient features, such as the generation of stable and scale invariant primordial density perturbations, absence of ghosts, and no violation of the null energy condition throughout the cosmic evolution [30]. It thus motivates us to investigate bounce universe models further and derive testable predictions in a model-independent manner. Dark matter creation and evolution is studied in the bounce universe [33-37]. When sufficient dark matter particles are not produced to reach kinematic equilibrium during contraction, relic abundance in the present universe then imposes a constraint on the dark matter mass and coupling constant. We can then produce numerical predictions for heavy dark matter in nuclear recoils experiments [35] and for light dark matter in electron-scattering experiments [37].

In the rest of this paper, after providing a short review of the CSTB model, we present a thorough study of the parameter space of this model. Our results verify that CSTB can produce a sufficient number of e-foldings to solve the problems related to flatness, horizon, homogeneity and Big Bang singularity, which will result in a universe of the current size.
Furthermore this is achieved with a large volume of the parameter space with no limitation on the values of the free parameters in the model. In other words the problems related to the Big Bang, including the Big Bang problem, are solved naturally in the CSTB model.

\section{The Single Bounce Criteria}

In this section, we review briefly the CSTB model and discuss the essential physics of this string cosmology model based on D-brane and $\bar{D}$-brane annihilations. In the effective theory, a D-D̄3-brane pair is described by the open string tachyon field action [28],

$$
\mathcal{L}_{T}=V_{T} \sqrt{1+M_{s}^{-4} \partial_{\mu} T \partial^{\mu} T}, V_{T}=\frac{V_{0}}{\cosh \left(\frac{T}{\sqrt{2} M_{s}}\right)}
$$

where $T, M_{s}, V_{0}$ are the tachyon field, the string mass, and the tension of D-D̄3-brane pair, respectively. The tachyon potential $V_{T}$ has a maximum at $T=0$ and two minima at $T \rightarrow \pm \infty$. We take the background metric as $(-,+,+,+)$, and we also assume that the tachyon field is spatially homogenous.

In D-brane inflation [38] the attractive potential of the D- $\bar{D}$ pair takes the following form:

$$
V_{\phi}=\frac{1}{2} m_{\phi}^{2} \phi^{2}+V_{0}-\frac{V_{0}^{2}}{4 \pi^{2} v \phi^{4}}, \quad \phi \equiv \sqrt{V_{0}} y,
$$

where $y$ being the distance between the D-D̄3-branes.

The CSTB model [30-32] introduces a scalar-tachyon coupling term $\lambda \phi^{2} T^{2}$, and the tachyon condensation comes about naturally after a period of the locked inflation, driven by the tension of D-D̄3-branes, and another period of rolling inflation while rolling down its potential hill. The coupling of between the scalar and the tachyon plays a crucial role in the cosmic evolution by greatly extending the time the tachyon spends at the top of its potential hill and hence increasing the number of the e-foldings obtained in the periods of locked inflation and rolling inflation. This is other impossible [27-29].

The CSTB Lagrangian takes the form:

$\mathcal{L}=\mathcal{L}_{T}-\frac{1}{2} \partial_{\mu} \phi \partial^{\mu} \phi-\frac{1}{2} m_{\phi}^{2} \phi^{2}-\lambda \phi^{2} T^{2}$,

in addition to the Hilbert-Einstein term, in a FRW background with $k=1$,

$d s^{2}=-d t^{2}+a^{2}(t)\left[\frac{d r^{2}}{1-k r^{2}}+r^{2}\left(d \theta^{2}+\sin ^{2} \theta d \phi^{2}\right)\right]$.

Since each component of this model has the correct sign of kinetic energy term and positive potential, we have $\rho_{\text {tot }}>0$ and $\omega_{t o t}=\frac{\rho_{t o t}}{p_{t o t}} \geqslant-1$, where $\omega_{t o t}, \rho_{t o t}$ and $p_{t o t}$ being, respectively, the Equation of State, energy density and pressure derived from the model. The CSTB universe model is thus free of ghosts and satisfies the null energy condition $p+\rho \geqslant 0$ throughout the cosmic evolution [39]. 


\subsection{Cosmic evolution in the CSTB model}

The CSTB cosmos starts at action at $C^{\prime}$ as it undergoes a period of tachyon matter dominated contraction, from $C^{\prime}$ to $B^{\prime}$, during which the tachyon behaves like cold dark matter $[28,40]$, From $B^{\prime}$ to $A$, it undergoes a period of accelerating contraction as the potential energy comes into play. A locked inflation era takes place from $A$ to $B$ (about the bounce point $\dot{a}=0$ and $\ddot{a}>0$ ) as the universe undergoes a period of exponential expansion driven by the vacuum energy of the tachyon (due to the brane tension). This period is extended by the effective coupling of tachyon and Higgs fields, and it solves the problems with tachyon inflation pointed out by [41]. Quantum mechanically the tachyon is prevented from decaying (tachyon condensation) by the fast oscillations of the Higgs fields. The tachyon condensation eventually takes place at $B$, where all the energy of tachyon field transfers to tachyon matter $[28,40]$. The phase transition at $B$ is followed by a tachyon matter dominated period, $B \rightarrow C$. At point $C^{\prime}$, reheating may happen as the universe undergoes another phase transition, during which the energy of tachyon matter transfers to radiation. This is depicted schematically in Fig.??.

The background cosmology evolves according to the Friedman equations,

$H^{2}=-\frac{1}{a^{2}}+\frac{8 \pi}{3 M_{p}^{2}}\left[\frac{V(T)}{\sqrt{1-M_{s}^{-4} \dot{T}^{2}}}+\left(\frac{1}{2} m_{\phi}^{2}+\lambda T^{2}\right) \phi^{2}+\frac{1}{2} \dot{\phi}^{2}\right] ;$

whereas the equations of motion for $T$ and $\phi$ are governed by

$$
\begin{array}{r}
\ddot{T}^{2}+\left(1-\dot{T}^{2}\right)\left[3 H \dot{T}+\frac{V^{\prime}(T)+2 \lambda \phi^{2} T \sqrt{1-\dot{T}^{2}}}{V(T)}\right]=0, \\
\ddot{\phi}+3 H \dot{\phi}+\left(m_{\phi}^{2}+2 \lambda T^{2}\right) \phi=0 ;
\end{array}
$$

with the string mass, $M_{s}$, being suppressed in the above equations.

The solutions to these equations of motion have been studied [30] in detail. It turns out, by introducing the coupling term $\lambda T^{2} \phi^{2}$, CSTB model suggests a novel feature: for a nonvanishing value of $\phi$, the coupling term pulls back the vacuum expectation value of this model at finite, $(T, \phi)=\left(T_{\mathrm{c}}, 0\right)$, as shown in Figure ?? and in [31,32]. In contrast to the single tachyon field model which tachyon keep rolling towards infinity [27], the tachyon in this model is stabilised around the effective vacuum with quasi-harmonic oscillations. This feature in turn pave the way for the studying phase transition and matter generation in the bounce universe context $[42,43]$.

A detailed analysis of the cosmological perturbations of this model has been carried in $[31,32]$. The spectrum of the primordial matter perturbations takes the form (c.f Eq.(3.18) in $[31,32])$ :

$P_{\zeta}=\left(\frac{H}{\dot{T}_{c}}\right)^{2} P_{\delta T}=\frac{C^{2}}{16 \pi \kappa} k^{0} \eta^{0}$

with $k$ being the wave vector and $\eta$ being the conformal time. $P_{\delta T}$ is the spectrum of tachyon field whose perturbations are responsible for primordial matter generation [31,32]. Both $C$ and $\kappa$ are constant parameters whose values are determined by $\lambda$ and $\frac{M_{s}}{M_{p}}$. Therefore, the primordial curvature perturbation of this model is scale invariant and stable. Furthermore, taking the sub-leading effects into account, the spectral index of the primordial curvature perturbation is also obtained in Eq.(3.22) of [31,32]. It is thus natural obtain the value of $n_{s}-1 \leqslant 0.04$ around a few percents in the CSTB model. A detailed analysis presented in [31,32] shows that a large parameter space in CSTB model satisfies the current observational constraints of spectral index.

\subsection{How to make a successful bounce: Initial conditions}

In this section we study the constraints on parameters from having enough e-foldings. In the locked inflation era the universe is dominated by vacuum energy. The Hubble parameter is nearly constant as long as the tachyon is locked at its peak by the scalar field $\phi$. The dynamic equations of the Hubble parameter, $\phi$ and $T$ can hence be simplified as follows,

$$
\begin{aligned}
H^{2}-\frac{8 \pi V_{0}}{3 M_{p}^{2}} & =0 \\
\ddot{\phi}+3 H \dot{\phi}+m_{\phi}^{2} \phi & =0 \\
\frac{1}{M_{s}^{4}} \ddot{T}+\left(-\frac{1}{2 M_{s}^{2}}+\frac{2 \lambda \phi^{2}}{V_{0}}\right) T & =0 .
\end{aligned}
$$

The scalar field, $\phi$, oscillates while being redshifted

$$
\phi \propto a^{-\frac{3}{2}} e^{i \sqrt{m_{\phi}^{2}-\frac{9}{4} H^{2}} t} .
$$

We can calculate the number of e-foldings $N_{L}$ in this era determined by the critical value of $\left\langle\phi_{c}^{2}\right\rangle=V_{0} / 4 \lambda M_{s}^{2}$ :

$N_{L}=\frac{1}{6} \ln \frac{32 \lambda^{3} M_{s}^{4}\left\langle\phi_{0}^{2}\right\rangle^{2}\left\langle T_{0}^{2}\right\rangle}{m^{2} V_{0}^{2}}$.

Let us now turn to observational constraints on CSTB model:

- A period of vacuum energy domination: The first constraint comes from vacuum energy domination. In the locked inflation era, the tension of D- $\overline{\mathrm{D}}$-brane is so large that it dominates the energy density in this era.

$V_{0}>\frac{1}{2} m^{2}\left\langle\phi_{0}^{2}\right\rangle+\lambda\left\langle T_{0}^{2}\right\rangle\left\langle\phi_{0}^{2}\right\rangle$ 
- The bounce point: For an extended period of locked inflation about $T \sim 0$, the initial value $\left\langle\phi_{0}^{2}\right\rangle$ should be larger than $\left\langle\phi_{1}^{2}\right\rangle$. And the locked inflation ends when

$\left\langle\phi_{0}^{2}\right\rangle<\frac{V_{0}}{4 \lambda M_{s}^{2}}$

- The effective mass of $\phi$ : The effective mass of $\phi$ is given by $m_{e f f}=m_{\phi}^{2}+2 \lambda T^{2}$. During locked inflation, $T$ red-shifts and its vev $\left\langle T^{2}\right\rangle$ diminishes dramatically. We assume that at the beginning of the locked inflation era $T_{0}$ is very large and that the $T$ field is smaller than the bare mass of scalar field, $m_{\phi}$ at the end. Therefore we have

$2 \lambda\left\langle T_{0}^{2}\right\rangle>m_{\phi}^{2}>2 \lambda\left\langle T_{1}^{2}\right\rangle$

and $\left\langle T_{1}^{2}\right\rangle$ can be expressed as $\sqrt{\frac{m_{\phi}^{2} V_{0}\left\langle T_{0}^{2}\right\rangle}{8 \lambda^{2} M_{s}^{2}\left\langle\phi_{0}^{2}\right\rangle}}$.

- Keeping $\phi$ oscillating: To keep $\phi$ oscillating in the locked inflation era, we demand the frequency of $\phi$ in 9 be real, which in turn implies $m_{\phi}^{2} \phi>\frac{9}{4} H^{2}$.

$2 \lambda\left\langle T_{0}^{2}\right\rangle>\frac{6 \pi}{M_{p}^{2}}\left(V_{0}+2 \lambda\left\langle T_{0}^{2}\right\rangle\left\langle\phi_{0}^{2}\right\rangle\right)$.

With these constraints we deduce two critical relations amongst the key parameters $m_{\phi}, M_{s}$ and $\lambda$ :

$$
\begin{aligned}
0<\frac{m}{\lambda M_{p}}<\frac{4(\sqrt{2}-1)}{\sqrt{3 \pi}} & \sim 0.54, \\
\lambda & >\frac{1}{8} .
\end{aligned}
$$

We thus see that these values cover a wide volume of the parameter space, and it is easy to pick a generic set of initial conditions for a successful bounce model resulting in a realistic universe. In particular this only requires that $N_{L} \sim 6$ e-foldings are generated in the locked inflation era. This is to be contrasted with the fine tuning one needs for successful inflation modelling.

\section{The contraction era in CSTB Universe}

We shall show that CSTB universe is free of the Horizon problem by calculating the e-foldings in the contraction phase of the CSTB universe.

\subsection{CSTB model solves the horizon problem}

Bounce universe models solve the horizon problem because there exists a phase of contraction-prior to the bounce-long enough to put the entire universe back into thermal contact and re-establish causality. In CSTB model, particle horizon is therefore nonzero. Considering that particle horizons in expansion and contraction phases are symmetric, we only calculate the particle horizon in expansion phase.

In the era of locked inflation, the universe is vacuum energy dominated: the Friedman equation becomes equation 6 and the universe undergoes an exponential expansion

$a=a_{0} e^{\sqrt{\frac{8 \pi V_{0}}{3 M_{p}^{2}}} t}$.

The particle horizon in locked inflation is given by

$d_{p_{1}}=a_{1} \int_{a_{0}}^{a_{1}} \frac{d a}{H a^{2}}=\frac{a_{1}}{H_{0}}\left(\frac{1}{a_{0}}-\frac{1}{a_{\max }}\right)$.

Shortly after the bounce the Hubble parameter changes from 0 to $\sqrt{\frac{8 \pi V_{0}}{3 M_{p}^{2}}}$, and $H_{0} a_{0}=1$, which in turn implies

$$
d_{p_{1}}=a_{1}-a_{0} .
$$

In the tachyon matter dominated era, the growth of the particle horizon is

$$
d_{p_{2}}=a_{\max } \int_{a_{1}}^{a_{\max }} \frac{d a}{H a^{2}}=\frac{2 a_{0} a_{\max }}{a_{1}^{\frac{3}{2}}}\left(\sqrt{a_{\max }}-\sqrt{a_{1}}\right) .
$$

All in all the total particle horizon at the end of expansion would be

$$
d_{p_{t o t}}=d_{p_{1}}+d_{p_{2}}=a_{1}-a_{0}+\frac{2 a_{0} a_{\max }}{a_{1}^{\frac{3}{2}}}\left(\sqrt{a_{\max }}-\sqrt{a_{1}}\right)
$$

and thus $\frac{d_{p_{t o t}}}{a_{0}} \gg 1$. Since the maximum size of universe is larger than current size $a_{0}$ at the bounce point the particle horizon is much larger than the scale factor. So bounce universe models guarantee that the particle horizon be larger than the current homogeneous regions.

\subsection{E-foldings of the universe}

In Big Bang cosmos the flatness problem is a cosmological fine-tuning problem. CMB data [3] determines the current universe to be flat up to $\sim 1 \%$ percent level, $\Omega=1.00 \pm 0.01$,

$$
\frac{1}{a_{\text {now }}^{2} H_{\text {now }}^{2}}<0.01 \text {. }
$$

As shown in Fig. ?? we can read off the required e-foldings from the end of locked inflation to the present:

$$
\begin{aligned}
N_{r}=\ln \left(\frac{a_{1}}{a_{\text {now }}}\right) & =\ln \left(\frac{\rho_{\text {now }}}{\rho_{D}}\right)^{\frac{1}{3}}\left(\frac{\rho_{D}}{\rho_{C}}\right)^{\frac{1}{4}}\left(\frac{\rho_{C}}{\rho_{B}}\right)^{\frac{1}{3}} \\
& =\ln \left(\frac{T_{\text {now }}}{T_{D}}\right)^{\frac{4}{3}}\left(\frac{T_{D}}{T_{C}}\right)\left(\frac{T_{C}}{T_{B}}\right)^{\frac{4}{3}} .
\end{aligned}
$$

After the tachyon condenses the single tachyon field rolls towards infinity with almost zero effective mass. When $\langle T\rangle$ becomes large, the tachyon field can be replaced by an equivalent scalar field $\sigma=\frac{4 \sqrt{V_{0}}}{M_{s}} e^{-\frac{T}{2 \sqrt{2} M_{s}}}$, which approaches zero 
when $T \rightarrow \infty$. With the new field the action can be expanded around $\sigma \sim 0$ and to first order in $\lambda$,

$L_{\phi-\sigma}=-\frac{1}{2} \sigma^{2}-\frac{1}{2} \partial_{\mu} \sigma \partial^{\mu} \sigma-\frac{1}{2} \partial_{\mu} \phi \partial^{\mu} \phi-\frac{1}{2} m^{2} \phi^{2}-\lambda \phi^{2} \sigma^{2}$,

i.e. at point $C^{\prime}$ in Fig. ??, with

$\left(\frac{\dot{a}}{a}\right)^{2}=-\frac{1}{a^{2}}+\frac{8 \pi}{3 M_{p}^{2}}\left[\frac{1}{2} \sigma^{2}+\frac{1}{2} m^{2} \phi^{2}-\lambda \sigma^{2} \phi^{2}+\frac{1}{2} \dot{\phi}^{2}+\frac{1}{2} \dot{\sigma}^{2}\right]$.

Scalar fields, $\sigma$ and $\phi$, then take on similar equations of motion:

$$
\begin{aligned}
\ddot{\sigma}+3 H \dot{\sigma}+\left[\left(\frac{M_{s}}{2}\right)^{2}-2 \lambda \phi^{2}\right] \sigma & =0 \\
\ddot{\phi}+3 H \dot{\phi}+\left(m^{2}-2 \lambda \sigma^{2}\right) \phi & =0 .
\end{aligned}
$$

From (21) we can deduce that at $C^{\prime},\left\langle\sigma^{2}\right\rangle$ and $\left\langle\phi^{2}\right\rangle$ are at their minima, and divide the energy density equally:

$\left\langle\phi^{2}\right\rangle_{\min }=\frac{3 M_{p}^{2}}{8 \pi m^{2} a^{2}}$.

During the subsequent contraction phase, as the scale factor $a$ decreases, $\left\langle\sigma^{2}\right\rangle$ and $\left\langle\phi^{2}\right\rangle$ blue-shift, leading to a decrease in the effective masses. Considering that $m_{\sigma}^{2}=\left(\frac{m_{s}}{2}\right)^{2}-2 \lambda \phi^{2}$, $m_{\sigma}^{2}$ decreases to zero and $\sigma$ loses its validity and one should returns to $T$. At $B^{\prime}$ in Figure ??, the contraction phase turns into deflation. We denote the amplitude of $\phi$ at this point by:

$\left\langle\phi^{2}\right\rangle_{e}=\frac{m_{s}^{2}}{8 \lambda}$.

E-foldings can be computed accordingly,

$$
N_{c}=\frac{1}{3} \ln \frac{\left\langle\phi^{2}\right\rangle_{e}}{\left\langle\phi^{2}\right\rangle_{\min }}
$$

because $\left\langle\phi^{2}\right\rangle \propto a^{3}$. Upon substituting the current size of universe $a_{\text {current }} \sim 5.4 \times 10^{61} l_{p}$ into $N_{c}$, we push back the current universe to the beginning of the deflation era:

$N_{c}=\frac{1}{3} \ln \frac{8 \pi m^{2} m_{s}^{2} a^{2}}{8 \lambda 3 M_{p}^{2}} \sim 118$,

where $m \sim \frac{1}{10} m_{s} \sim \frac{1}{100} M_{p}, \lambda \sim 0.25$. It is persuasive to say that, with a contraction which can generate 120 e-foldings, the expansion era after the bounce can enjoy an analogous number of e-foldings [10]. We can thus conclude that CSTB cosmology model solves the flatness problem by the 118 efoldings in the contraction phase.

\section{Conclusion and outlook}

We present a viable alternative to inflation based on string theory utilising coupled scalar and tachyon fields in the DD-brane system [30]. The CSTB cosmos undergoes a period of tachyon matter dominated contraction in which a stable and scale invariant spectrum of primordial density perturbations is produced $[31,32]$. The model has a signature out-ofthermal-equilibrium production of dark matter which can be tested by the future array of dark matter detections [33-37], independently of cosmological observations [44]. Exploration on matter production and baryon asymmetry genesis is well underway $[42,43]$. We expect our success can inspire further studies on bounce models built from string theory or other quantum gravity theories.

Recently an interesting attempt is made to use AdS/CFT correspondence to study the evolution of matter through the bounce point [46] which is inspired by an earlier attempt of using AdS/CFT to study cosmic singularity in the bound universe [45]. Pioneer works on studying AdS singularity can be found in [47-54], together with earlier initiatives of using AdS/CFT to study cosmic singularities [55-66]. A thorough study of physics in the bounce universe is in sight.

Another interesting question to ask if the CST Bounce universe model can be imbedded in higher dimensions, a la [67], and to study the stabilisation of the extra moduli in this context. Further explorations shall also exploit the symmetries and dualities of the mother string theory as string-cosmology models utilising T-duality $[68,69]$. This direction is expected to hold more promise of modulus stabilisation [70-72].

We would like to thank Jin U Kang, Changhong Li and Yuan Xin for useful discussions. This research project has been supported in parts by the NSF China under Contract No. 11775110 and No. 11690034. We also acknowledge the European Union's Horizon 2020 Research and Innovation (RISE) programme under the Marie Sklodowska-Curie grant agreement No. 644121, and the Priority Academic Program Development for Jiangsu Higher Education Institutions (PAPD).

1 Guth, Alan H. "Inflationary universe: A possible solution to the horizon and flatness problems." Physical Review D 23.2 (1981): 347;

2 A. H. Guth, D. I. Kaiser and Y. Nomura, "Inflationary paradigm after Planck 2013," Phys. Lett. B 733, 112 (2014) doi:10.1016/j.physletb.2014.03.020 [arXiv:1312.7619 [astro-ph.CO]]; A. Linde, "Inflationary Cosmology after Planck 2013," doi:10.1093/acprof:oso/9780198728856.003.0006 arXiv:1402.0526 [hep-th];

3 G. Hinshaw et al. [WMAP Collaboration], "Nine-Year Wilkinson Microwave Anisotropy Probe (WMAP) Observations: Cosmological Parameter Results," Astrophys. J. Suppl. 208, 19 (2013) [arXiv:1212.5226 [astro-ph.CO]]. P. A. R. Ade et al. [Planck Collaboration], "Planck 2015 results. XVI. Isotropy and statistics of the CMB," arXiv:1506.07135 [astro-ph.CO].

4 Mukhanov, Viatcheslav F. 'Quantum fluctuation and 'nonsingular' universe". JETP Lett. 33: 532 4 ? (1981); "The vacuum energy and large scale structure of the universe". Sov. Phys. JETP 56: 258ф?5.

5 A. Borde and A. Vilenkin, "Eternal inflation and the initial singularity," Phys. Rev. Lett. 72 (1994) 3305 doi:10.1103/PhysRevLett.72.3305 [grqc/9312022].

6 Trodden, M., V. F. Mukhanov, and Robert H. Brandenberger. "A nonsingular two dimensional black hole.” Phys Lett B 316.4 (1993): 483487. 
7 Gasperini, Maurizio, and Gabriele Veneziano. "The pre-big bang scenario in string cosmology." Physics Reports 373.1 (2003): 1-212.

8 A. Linde, "Inflation and string cosmology," Fortsch. Phys. 57, 418 (2009). doi:10.1002/prop.200900054

9 S.-H. Henry Tye, "Brane inflation: String theory viewed from the cosmos," Lect. Notes Phys. 737, 949 (2008) [hep-th/0610221].

10 S. Nojiri, S. D. Odintsov and V. K. Oikonomou, "Modified Gravity Theories on a Nutshell: Inflation, Bounce and Late-time Evolution," Phys. Rept. 692, 1 (2017) doi:10.1016/j.physrep.2017.06.001 [arXiv:1705.11098 [gr-qc]].

R. Brandenberger and P. Peter, "Bouncing Cosmologies: Progress and Problems," arXiv:1603.05834 [hep-th].

D. Battefeld and P. Peter, "A Critical Review of Classical Bouncing Cosmologies," Phys. Rept. 571, 1 (2015) doi:10.1016/j.physrep.2014.12.004 [arXiv:1406.2790 [astro-ph.CO]]. E. Wilson-Ewing, "The Matter Bounce Scenario in Loop Quantum Cosmology," JCAP 1303, 026 (2013) doi:10.1088/14757516/2013/03/026 [arXiv:1211.6269 [gr-qc]]

M. Novello and S. E. P. Bergliaffa, "Bouncing Cosmologies," Phys. Rept. 463, 127 (2008) [arXiv:0802.1634 [astro-ph]]. Y. F. Cai, D. A. Easson and R. Brandenberger, "Towards a Nonsingular Bouncing Cosmology," JCAP 1208, 020 (2012) doi:10.1088/14757516/2012/08/020 [arXiv:1206.2382 [hep-th]].

11 P. J. Steinhardt and N. Turok, "A Cyclic model of the universe," hepth/0111030.

12 D. Wands, "Duality invariance of cosmological perturbation spectra," Phys. Rev. D 60, 023507 (1999) doi:10.1103/PhysRevD.60.023507 [gr-qc/9809062].

13 F. Finelli and R. Brandenberger, "On the generation of a scale-invariant spectrum of adiabatic fluctuations in cosmological models with a contracting phase," Phys. Rev. D 65, 103522 (2002) [arXiv:hepth/0112249].

14 Lin, Chunshan, Robert H. Brandenberger, and Laurence Perreault Levasseur. "A matter bounce by means of ghost condensation." JCAP 2011.04 (2011): 019;

Brandenberger, Robert H. "The matter bounce alternative to inflationary cosmology.” arXiv preprint arXiv:1206.4196 (2012).

15 R. Brandenberger, "Matter Bounce in Horava-Lifshitz Cosmology," Phys. Rev. D 80, 043516 (2009) doi:10.1103/PhysRevD.80.043516 [arXiv:0904.2835 [hep-th]]

16 T. Qiu and K. C. Yang, "Perturbations in Matter Bounce with Non-minimal Coupling," JCAP 1011, 012 (2010) doi:10.1088/14757516/2010/11/012 [arXiv:1007.2571 [astro-ph.CO]].

17 Y. F. Cai, S. H. Chen, J. B. Dent, S. Dutta and E. N. Saridakis, "Matter Bounce Cosmology with the $\mathrm{f}(\mathrm{T})$ Gravity," Class. Quant. Grav. 28, 215011 (2011) doi:10.1088/0264-9381/28/21/215011 [arXiv:1104.4349 [astro-ph.CO]].

18 S. D. Odintsov and V. K. Oikonomou, "Matter Bounce Loop Quantum Cosmology from $F(R)$ Gravity," Phys. Rev. D 90, no. 12, 124083 (2014) doi:10.1103/PhysRevD.90.124083 [arXiv:1410.8183 [gr-qc]].

19 Koehn, Michael, Jean-Luc Lehners, and Burt A. Ovrut. "Cosmological super-bounce.” Physical Review D 90.2 (2014): 025005.

20 Qiu, Taotao, and Yu-Tong Wang. "G-Bounce Inflation: Towards Nonsingular Inflation Cosmology with Galileon Field.” arXiv:1501.03568 (2015).

21 Bhattacharya, Kaushik, Yi-Fu Cai, and Suratna Das. "Lee-Wick radiation induced bouncing universe models." Physical Review D 87.8 (2013): 083511;

Cai, Yi-Fu, et al. "Nonsingular cosmology with a scale-invariant spectrum of cosmological perturbations from Lee-Wick theory." Physical Review D 80.2 (2009): 023511;

22 Y. F. Cai, T. t. Qiu, R. Brandenberger and X. m. Zhang, "A Nonsingular Cosmology with a Scale-Invariant Spectrum of Cosmological Perturbations from Lee-Wick Theory," Phys. Rev. D 80 (2009) 023511 doi:10.1103/PhysRevD.80.023511 [arXiv:0810.4677 [hep-th]].
23 Cai, Yi-Fu, et al. "Bouncing universe with quintom matter." Journal of High Energy Physics 2007.10 (2007): 071;

24 Cai, Yi-Fu, and Xinmin Zhang. "Evolution of metric perturbations in quintom bounce model.” arXiv:0808.2551 (2008).

25 J. Khoury, B. A. Ovrut, P. J. Steinhardt and N. Turok, "The Ekpyrotic universe: Colliding branes and the origin of the hot big bang," Phys. Rev. D 64, 123522 (2001) doi:10.1103/PhysRevD.64.123522 [hepth/0103239]. D. H. Lyth, "The Primordial curvature perturbation in the ekpyrotic universe," Phys. Lett. B 524, 1 (2002) doi:10.1016/S03702693(01)01374-0 [hep-ph/0106153]. R. Brandenberger and F. Finelli, "On the spectrum of fluctuations in an effective field theory of the Ekpyrotic universe," JHEP 0111, 056 (2001) doi:10.1088/11266708/2001/11/056 [hep-th/0109004].

26 S. Carloni, P. K. S. Dunsby and D. M. Solomons, "Bounce conditions in $\mathrm{f}(\mathrm{R})$ cosmologies," Class. Quant. Grav. 23, 1913 (2006) doi:10.1088/0264-9381/23/6/006 [gr-qc/0510130].

27 A. Sen, "Rolling tachyon," JHEP 0204, 048 (2002) doi:10.1088/11266708/2002/04/048 [hep-th/0203211].

28 A. Sen, "Tachyon matter," JHEP 0207, 065 (2002) doi:10.1088/11266708/2002/07/065 [hep-th/0203265].

29 G. W. Gibbons, "Cosmological evolution of the rolling tachyon," Phys. Lett. B 537, 1 (2002) doi:10.1016/S0370-2693(02)01881-6 [hepth/0204008].

30 C. Li, L. Wang and Y. K. E. Cheung, "Bound to bounce: A coupled scalar фtachyon model for a smooth bouncing/cyclic universe," Phys. Dark Univ. 3, 18 (2014) [arXiv:1101.0202 [gr-qc]].

31 C. Li and Y. K. E. Cheung, "Dualities between Scale Invariant and Magnitude Invariant Perturbation Spectra in Inflationary/Bouncing Cosmos," arXiv:1211.1610 [gr-qc].

32 C. Li and Y. K. E. Cheung, "The scale invariant power spectrum of the primordial curvature perturbations from the coupled scalar tachyon bounce cosmos," JCAP 1407, 008 (2014) doi:10.1088/14757516/2014/07/008 [arXiv:1401.0094 [gr-qc]].

33 C. Li, R. H. Brandenberger and Y. K. E. Cheung, "Big Bounce Genesis," Phys. Rev. D 90, no. 12, 123535 (2014) [arXiv:1403.5625 [grqc]].

34 Y. K. E. Cheung, J. U. Kang and C. Li, "Dark matter in a bouncing universe," JCAP 1411, no. 11, 001 (2014) [arXiv:1408.4387 [astroph.CO]].

35 Y. K. E. Cheung and J. D. Vergados, "Direct dark matter searches Test of the Big Bounce Cosmology," JCAP 1502, no. 02, 014 (2015) [arXiv:1410.5710 [hep-ph]].

36 Y. K. E. Cheung, C. Li and J. D. Vergados, "Big Bounce Genesis and Possible Experimental Tests - A Brief Review," arXiv:1611.04027 [astro-ph.CO].

37 J. D. Vergados, C. C. Moustakidis, Y. K. E. Cheung, H. Ejri, Y. Kim and Y. Lie, "Light WIMP searches involving electron scattering," arXiv:1605.05413 [hep-ph].

38 Gia Dvali and S.-H. Henry Tye. "Brane inflation." Physics Letters B 450.1 (1999): 72-82.

39 C. Molina-Paris and M. Visser, "Minimal conditions for the creation of a Friedman-Robertson-Walker universe from a 'bounce',' Phys. Lett. B 455, 90 (1999) [arXiv:gr-qc/9810023].

40 A. Sen and B. Zwiebach, "Tachyon condensation in string field theory." Journal of High Energy Physics 2000.03 (2000): 002; N. Berkovits, A. Sen and B. Zwiebach, "Tachyon condensation in superstring field theory." Nuclear Physics B 587.1-3 (2000): 147-178.

41 L. Kofman and A. D. Linde, "Problems with tachyon inflation," JHEP 0207, 004 (2002) [hep-th/0205121].

42 M. Drewes and J. U. Kang, "Sterile neutrino Dark Matter production from scalar decay in a thermal bath," arXiv:1510.05646 [hep-ph];

Y. K. E. Cheung, M. Drewes, J. U. Kang and J. C. Kim, "Effective Action for Cosmological Scalar Fields at Finite Temperature," JHEP 1508, 059 (2015) doi:10.1007/JHEP08(2015)059 [arXiv:1504.04444 [hep-ph]]; 
Y.-K.E. Cheung and J. U Kang, "An alternative to reheating in a bounce universe" in progress.

43 Y.-K.E. Cheung, H.Q. Liu, M. J. Ramsey-Musolf, Y. Xin, "First Order Phase Transition for Baryogenesis in a Bounce Universe" to appear.

44 Y. F. Cai, Sci. China Phys. Mech. Astron. 57, 1414 (2014) doi:10.1007/s11433-014-5512-3 [arXiv:1405.1369 [hep-th]].

45 R. H. Brandenberger, Y. F. Cai, S. R. Das, E. G. M. Ferreira, I. A. Morrison and Y. Wang, "Fluctuations in a Cosmology with a Space-Like Singularity and their Gauge Theory Dual Description," arXiv:1601.00231 [hep-th].

46 L. Ming, T. Zheng and Y. K. E. Cheung, "Following the density perturbations through a bounce with AdS/CFT Correspondence," arXiv:1701.04287 [hep-th].

47 S. P. Kumar and V. Vaganov, "Quasinormal modes and holographic correlators in a crunching AdS geometry," arXiv:1512.07184 [hep-th].

48 A. Bzowski, T. Hertog and M. Schillo, "Cosmological singularities encoded in IR boundary correlations," arXiv:1512.05761 [hep-th].

49 S. P. Kumar and V. Vaganov, "Probing crunching AdS cosmologies," arXiv: 1510.03281 [hep-th].

50 J. L. F. Barbon and E. Rabinovici, "Holographic Complexity And Cosmological Singularities," arXiv:1509.09291 [hep-th].

51 N. Engelhardt and G. T. Horowitz, "Holographic Consequences of a No Transmission Principle," arXiv:1509.07509 [hep-th].

52 N. Engelhardt, T. Hertog and G. T. Horowitz, "Further Holographic Investigations of Big Bang Singularities," JHEP 1507 (2015) 044 doi:10.1007/JHEP07(2015)044 [arXiv:1503.08838 [hep-th]].

53 A. Enciso and N. Kamran, "Determining an asymptotically AdS Einstein spacetime from data on its conformal boundary," Gen. Rel. Grav. 47 (2015) 12, 147 doi:10.1007/s10714-015-1974-5 [arXiv:1502.01622 [gr-qc]].

54 S. Banerjee, S. Bhowmick, S. Chatterjee and S. Mukherji, "A note on AdS cosmology and gauge theory correlator," JHEP 1506 (2015) 043 doi:10.1007/JHEP06(2015)043 [arXiv:1501.06317 [hep-th]].

55 V. Balasubramanian, P. Kraus, A. E. Lawrence and S. P. Trivedi, Phys. Rev. D 59 (1999) 104021 doi:10.1103/PhysRevD.59.104021 [hepth/9808017].

56 T. Hertog and G. T. Horowitz, "Holographic description of AdS cosmologies," JHEP 0504 (2005) 005 doi:10.1088/11266708/2005/04/005 [hep-th/0503071].

57 A. Hamilton, D. N. Kabat, G. Lifschytz and D. A. Lowe, "Local bulk operators in AdS/CFT: A Boundary view of horizons and locality," Phys. Rev. D 73 (2006) 086003 doi:10.1103/PhysRevD.73.086003 [hep-th/0506118].
58 C. S. Chu and P. M. Ho, "Time-dependent AdS/CFT duality and null singularity," JHEP 0604 (2006) 013 doi:10.1088/11266708/2006/04/013 [hep-th/0602054].

59 S. R. Das, J. Michelson, K. Narayan and S. P. Trivedi, "Time dependent cosmologies and their duals," Phys. Rev. D 74 (2006) 026002 doi:10.1103/PhysRevD.74.026002 [hep-th/0602107].

60 C. S. Chu and P. M. Ho, "Time-dependent AdS/CFT duality. II. Holographic reconstruction of bulk metric and possible resolution of singularity," JHEP 0802 (2008) 058 doi:10.1088/1126-6708/2008/02/058 [arXiv:0710.2640 [hep-th]]

61 N. Turok, B. Craps and T. Hertog, "From big crunch to big bang with AdS/CFT," arXiv:0711.1824 [hep-th].

62 A. Awad, S. R. Das, K. Narayan and S. P. Trivedi, "Gauge theory duals of cosmological backgrounds and their energy momentum tensors," Phys. Rev. D 77 (2008) 046008 doi:10.1103/PhysRevD.77.046008 [arXiv:0711.2994 [hep-th]]

63 B. Craps, T. Hertog and N. Turok, "On the Quantum Resolution of Cosmological Singularities using AdS/CFT," Phys. Rev. D 86 (2012) 043513 doi:10.1103/PhysRevD.86.043513 [arXiv:0712.4180 [hep-th]].

64 J. L. F. Barbon and E. Rabinovici, "AdS Crunches, CFT Falls And Cosmological Complementarity," JHEP 1104 (2011) 044 doi:10.1007/JHEP04(2011)044 [arXiv:1102.3015 [hep-th]].

65 A. Enciso and N. Kamran, "Causality and the conformal boundary of AdS in real-time holography," Phys. Rev. D 85 (2012) 106016 doi:10.1103/PhysRevD.85.106016 [arXiv:1203.2743 [math-ph]].

66 N. Engelhardt, T. Hertog and G. T. Horowitz, "Holographic Signatures of Cosmological Singularities," Phys. Rev. Lett. 113 (2014) 121602 doi:10.1103/PhysRevLett.113.121602 [arXiv:1404.2309 [hep-th]].

67 L. Randall and R. Sundrum, "An Alternative to compactification," Phys. Rev. Lett. 83, 4690 (1999) [hep-th/9906064].

68 R. H. Brandenberger and C. Vafa, "Superstrings in the Early Universe," Nucl. Phys. B 316, 391 (1989).

69 C. Kounnas, H. Partouche and N. Toumbas, "Thermal duality and nonsingular cosmology in d-dimensional superstrings," Nucl. Phys. B 855, 280 (2012) [arXiv:1106.0946 [hep-th]].

70 A. J. Berndsen and J. M. Cline, "Dilaton stabilization in brane gas cosmology," Int. J. Mod. Phys. A 19, 5311 (2004) [hep-th/0408185].

71 S. Watson, "Moduli stabilization with the string Higgs effect," Phys. Rev. D 70, 066005 (2004) [hep-th/0404177].

72 R. Brandenberger, Y. K. E. Cheung and S. Watson, "Moduli stabilization with string gases and fluxes," JHEP 0605, 025 (2006) [hepth/0501032]. 


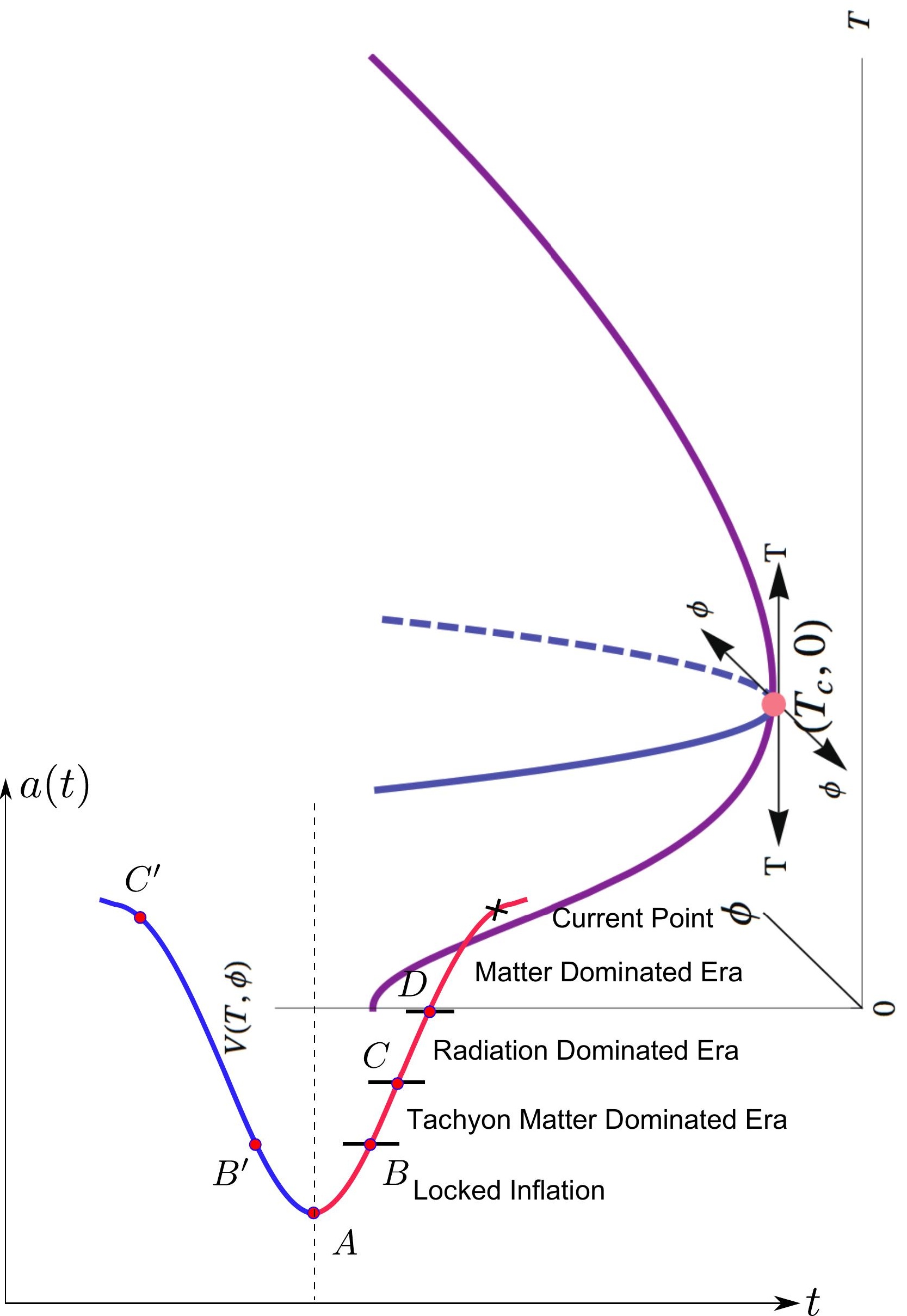




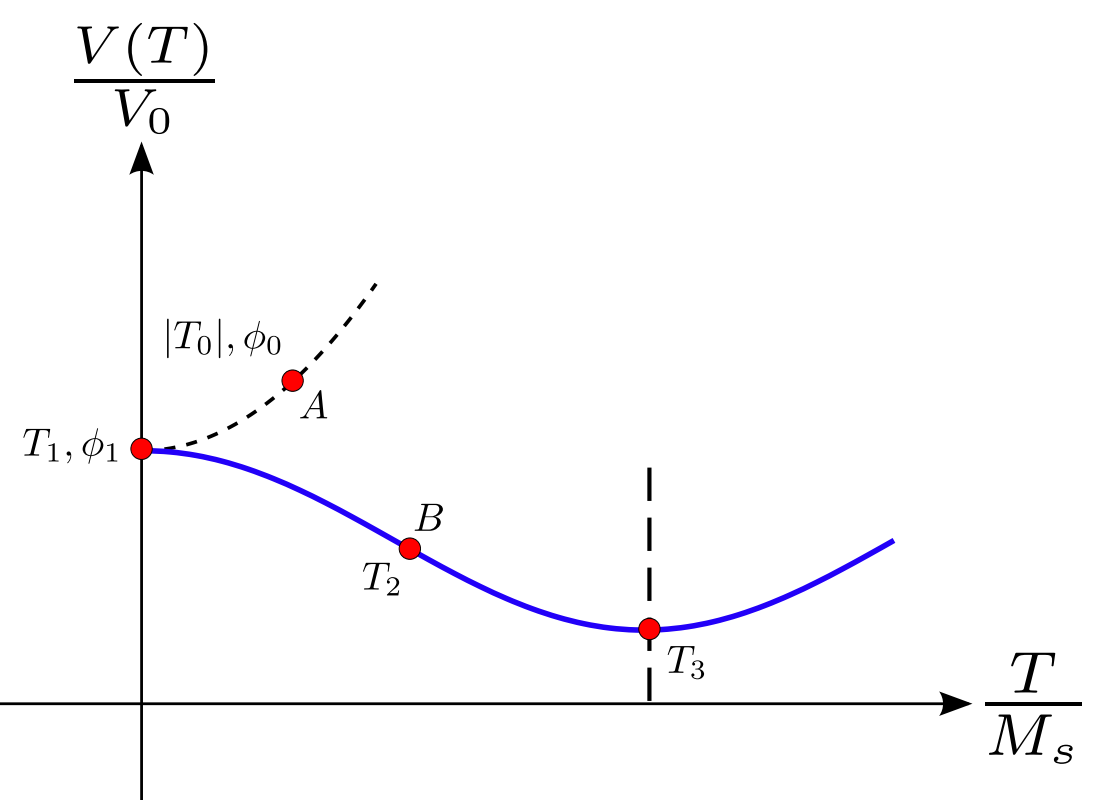

